\title{
OBTAINING PARTON DISTRIBUTION FUNCTIONS FROM SELF-ORGANIZING MAPS*
}

\author{
H. HONKANEN ${ }^{\dagger}$ AND S. LIUTI ${ }^{\ddagger}$ \\ Physics Department \\ 382 McCormick Rd., University of Virginia \\ Charlottesville, Virginia 22904, USA \\ Y.C. LOITIERE $\$$ D. BROGAN ${ }^{\top}$ AND P. REYNOLDS" \\ Department of Computer Science \\ School of Engineering, University of Virginia \\ 151 Engineer's Way, P.O. Box 400740 \\ Charlottesville, Virginia 22904 USA
}

\begin{abstract}
We present an alternative algorithm to global fitting procedures to construct Parton Distribution Functions parametrizations. The proposed algorithm uses SelfOrganizing Maps which at variance with the standard Neural Networks, are based on competitive-learning. Self-Organizing Maps generate a non-uniform projection from a high dimensional data space onto a low dimensional one (usually 1 or 2 dimensions) by clustering similar PDF representations together. The SOMs are trained on progressively narrower selections of data samples. The selection criterion is that of convergence towards a neighborhood of the experimental data. All available data sets on deep inelastic scattering in the kinematical region of $0.001 \leq x \leq 0.75$, and $1 \leq Q^{2} \leq 100 \mathrm{GeV}^{2}$, with a cut on the final state invariant mass, $W^{2} \geq 10 \mathrm{GeV}^{2}$ were implemented. The proposed fitting procedure, at variance with standard neural network approaches, allows for an increased control of the systematic bias by enabling the user to directly control the data selection procedure at various stages of the process.
\end{abstract}

\footnotetext{
*Work supported by grants 0426971 of the US National Science Foundation and and DE-FG02-01ER41200 of the US Department of Energy.

†hh9e@virginia.edu

¥sl4y@virginia.edu

§ycl2r@virginia.edu

ฯdbrogan@virginia.edu

" reynolds@virginia.edu
} 


\section{Introduction}

Parton Distribution Functions (PDFs) are defined as the probabilities to find a parton - a quark, antiquark or a gluon - of type $a$ in the proton with a given value of the process' scale defined by $Q^{2}$, the four-momentum transfer squared, and Bjorken's variable, $x_{B j}=Q^{2} / 2 M \nu, \nu$ being the energy transfer and $M$ the proton mass. $x_{B j}$ represents the light-cone momentum fraction of the proton carried by the parton. Although PDFs were studied both theoretically and experimentally for the past few decades, their determination is still hampered by a number of unsolved questions mainly concerning their Perturbative QCD (PQCD) evolution and, related to this, the treatment of heavy flavor quarks. Furthermore, this situation in particular the large indetermination of the gluon distribution - will have practical critical consequences on the predictivity of results at the LHC. PDFs were, in fact, recently defined as "a necessary evil" ${ }^{1}$. Our work was indeed motivated by similar concerns as the ones expressed in ${ }^{1}$.

To date, a few approaches have been developed that deal with the question of a fully quantitative determination of PDFs in a wide range of $x_{B j}$ and $Q^{2}$. On one side we have Global Fitting (GF) procedures, pursued, developed and refined since the beginning of QCD. ${ }^{\text {a }}$ More recently, a number of alternative approaches to GF were pursued, the main ones being the Neural Network (NN) approach ${ }^{3}$, and the Bayesian methods ${ }^{4}$. In both Refs. $^{3,4}$, the authors are concerned with the definition and evaluation of the PDFs uncertainties from GF. In particular, the $\chi^{2}$ obtained from the GF procedure is most likely to underestimate both the theoretical and experimental errors from the various data sets as proven by the existence of often large discrepancies in the results obtained by different groups ${ }^{1}$. In Ref. ${ }^{3}$, in particular, the main source of indetermination is attributed to the theoretical bias introduced by the choice of parametrization form of PDFs at the initial scale, $Q_{o}^{2}$, of PQCD evolution. However, implicit in NN algorithms is a hardly controllable systematic bias. The approach we propose here is based on a specific class of neural network algorithms, the Self-Organizing Maps (SOMs) (for a review see ${ }^{5}$ ). SOMs allow for a better control of the systematic bias by allowing to replace the fully automated procedure of standard NNs with an interactive fitting procedure, at the expense of re-introducing some theoretical bias in the fit. Our fitting procedure is based on an iterative process in which the "user" interactively delineates

${ }^{a}$ All results by the active groups in recent years are listed in ${ }^{2}$, and are also reported regularly at this conference. 
the boundary between acceptable and unacceptable results. Observables are clustered into a SOM and judged by the "user". A statistical analysis of the corresponding initial-scale PDFs is performed and gives rise to the next iteration of PDFs. Several criteria can be chosen by the user: from the minimization of $\chi^{2}$, to satisfying different sum rules, to selection on the behavior at low or large $x_{B j}$, etc.... In this contribution we show results based on the criterion of minimization of $\chi^{2}$ that allows us to gauge and test our initial results with the previously existing ones ${ }^{2}$.

\section{Method}

SOMs, at variance with standard NNs, are based on competitive-learning 5. In competitive learning one defines a number of "filters" that respond differently to the initial inputs in such a way that one or few of the filters are "winners" producing a high output. The "winners" create negative feedback so that only they and their neighbours get reinforced through the various cycles, or in other words, they get updated in learning. More technically, a SOM is an algorithm that maps in a topologically ordered way the training data onto a neural network. The mapping proceeds by selecting the neuron, $N_{W}$, that best matches each data sample according to a metric, $M_{D}$. Each neuron is represented in a two-dimensional grid, with coordinates: $\mathbf{x}_{\mathbf{i}} \equiv\left(x_{1}, x_{2}\right)$. A weighted average of each neuron, $N_{i}$ in the grid to the data sample is then performed, where the weight, $w_{i}$ is computed from the distance of $N_{i}$ to $N_{W}$ according to a metric, $M_{G}$, and a given neighborhood radius. $M_{G}$ defines the topology of the grid. This procedure is iterated with smaller radii until it saturates.

For our specific problem, the neurons correspond to the PDFs; the data are "synthetic data" (randomized samples of the original data). The metric $M_{G}$ that defines the topology of the map is:

$$
L_{1}(\mathbf{x}, \mathbf{y})=\sum_{j=1,2}\left|x_{j}-y_{j}\right|
$$

An important aspect of our procedure is that PQCD evolution is considered at every step. Our preliminary results are displayed in Fig.1 showing that our algorithm represents indeed a robust method to determine both the structure function $F_{2}\left(x_{B j}, Q^{2}\right)$, and the gluon distribution, $G\left(x_{B j}, Q^{2}\right)$, evolved at $Q^{2}=28.7 \mathrm{GeV}^{2}$.

We conclude that the proposed SOMPDFs, introudce a change of criteria with respect to NNPDFs aimed at bringing "theory" back in the loop, at variance with seeking full automation of the fitting procedure. They are 
$\mathrm{F}^{2}$ vs $\mathrm{X}$ at $\mathrm{Q}^{2}=28.7$

with 1-sigma error band

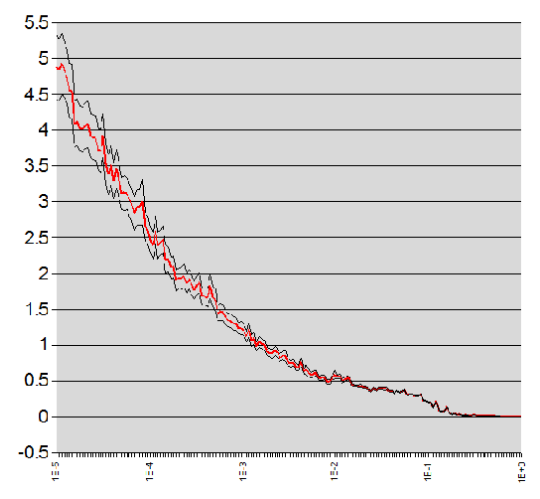

$F^{2}$ vs $X$ at $Q^{2}=207.4$

with 1-sigma error band

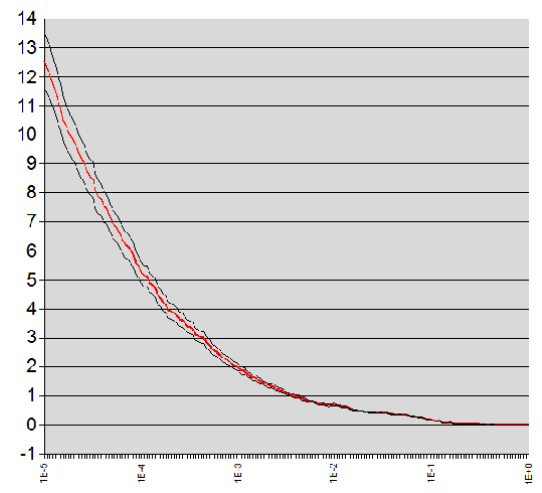

Figure 1. Left: Structure function $F_{2}\left(x, Q^{2}\right)$ from SOMPDFs fit, plotted vs. $x$ in the range $10^{-5}<x<1$, at $Q^{2}=28.7 \mathrm{GeV}^{2}$; Right: $F_{2}\left(x, Q^{2}\right)$ in the same range of $x$, at $Q^{2}=207 \mathrm{GeV}^{2}$.

therefore placed at the intersection between traditional GF methods and NN approaches. SOMPDFs have the following additional advantages over generic Genetic Algorithms that might help in future work to identify the role of different parameters: i) Visualization; ii) Dimensionality reduction; iii) Clustering (a study is on its way to determine what features of PDFs produce given patterns of clustering). We hope as future practical goals, to extend our investigation to addtional "filters" other than the $\chi^{2}{ }^{6}$, and to study the implementation of SOMPDFs in actual data analyses at the 
LHC using both nucleon and nuclear data.

\section{References}

1. J. Pumplin, AIP Conf. Proc. 792, 50 (2005)

2. http://durpdg.dur.ac.uk/HEPDATA/

3. L. Del Debbio, S. Forte, J. I. Latorre, A. Piccione and J. Rojo [NNPDF Collaboration], JHEP 0503, 080 (2005), and references therein; A. Piccione, these proceedings.

4. W. T. Giele, S. A. Keller and D. A. Kosower, arXiv:hep-ph/0104052; G. Cowan, these proceedings.

5. T. Kohonen, "Self Organizing Maps", Springer-Verlag, 1997.

6. H. Honkanen, S. Liuti, Y. Loitiere, D. Brogan and P. Reynolds, in preparation. 